\title{
Perturbations with angular momentum of Robinson-Trautman spacetimes
}

\author{
Osvaldo M. Moreschi ${ }^{1 * \dagger}$ and Alejandro Pérez ${ }^{2 \ddagger \S}$ \\ ${ }^{1}$ FaMAF, Universidad Nacional de Córdoba \\ Ciudad Universitaria, (5000) Córdoba, Argentina. \\ 2 Department of Physics and Astronomy, University of Pittsburgh, \\ 100 Allen Hall, Pittsburgh, PA 15260, USA.
}

March 8, 2001

\begin{abstract}
We study the possible asymptotically flat perturbations of Robinson-Trautman spacetimes. We differentiate between algebraically special perturbations and general perturbations. The equations that determine physically realistic spacetimes with angular momentum are presented.
\end{abstract}

PACS numbers: 04.30.Db, 04.25.-g, 04.70.Bw, 04.20.Jb

\section{Introduction}

Robinson-Trautman [17] spacetimes (RT) have been very useful for estimating the total gravitational radiation in the head-on black hole collision [13] [12] [2]. In reference [13] we have applied these geometries to the description of the total energy radiated in the head-on black hole collision with equal mass; and it was shown that our calculations agree remarkably well with the numerical exact calculations of Anninos et.al.[2]. The case of unequal mass black hole collision, was treated numerically in reference [1]; and our technique based on the use of the RT geometries [12] showed again an impressive agreement with the exact calculations. If one wants to generalize these estimates to the case of the black hole collision with orbital angular momentum it is necessary to consider spacetimes with total angular momentum. This is the main physical motivation for this work; the application of these calculations to specific physical models will be presented elsewhere 15.

\footnotetext{
${ }^{*}$ Member of CONICET.

${ }^{\dagger}$ Email: moreschi@fis.uncor.edu

${ }^{\ddagger}$ present address: Centre de Physique Théorique, CNRS Luminy, F-13288 Marseille, France.

$\S$ Andrew Mellon Predoctoral Fellowship holder.
} 
The Robinson-Trautman vacuum solutions are algebraically special spacetimes which are characterized by the existence of a congruence of diverging null geodesics without shear and twist. This implies the existence of a prefered family of null hypersurfaces; which provides a set of sections at future null infinity. The angular momentum calculated on these sections is found to be zero. (In a Bondi frame the asymptotic NP quantities $\Psi_{1}^{0}$ and $\sigma_{0}$ are zero; as a consequence the angular momentum vanishes independently of the definition used. See for example reference [9] and references therein.) In section 2 a we present a short review of the RT vacuum solutions.

It is natural then to consider the algebraically special perturbations of RT spacetimes; since they contain the RT family in the limit when the twist goes to zero.

Algebraically special spacetimes are physically very important solutions since they contain the RT family, the Kerr family of solutions and so also the Schwarzschild solution. In section 3 we present in full detail the algebraically special vacuum solutions in terms of a new frame, the so called 'standard rotating null tetrad' [16].

The algebraically special perturbations of RT spacetimes are governed by rather complicated equations; however, the equations simplify when one analysis the geometry in the far future in the limit of the asymptotic retarded time going to infinity. This is due to the fact that the RT geometry simplifies considerably in this limit. It will be shown that the total angular momentum of these perturbations is constant in the asymptotic future; therefore these spacetimes are not suitable for the description of a perturbed black hole with a dynamic angular momentum. Furthermore, the general algebraically special perturbations of RT spacetimes turn out to be divergent in the asymptotic future. These characteristics do not seem to be appropriate for the modeling of a spacetime representing the last stage after the collision of two compact objects with non-zero total angular momentum. We are therefore forced to consider general perturbations of RT spacetimes.

We dedicate section 4 to the presentation of general perturbations of RT spacetimes. The radial dependence of the tetrad is explicitly solved; and we also present the intrinsic evolution equations at future null infinity, which are used in the calculation of gravitational radiation.

The important issue of gauge freedom is discussed in both cases, algebraically special spacetimes (section 3) and general perturbation of RT spacetimes (section (4).

Summarizing comments are included in the last section 5 .

Throughout the work we will use the GHP [7] notation.

\section{Robinson-Trautman spacetimes}

Robinson and Trautman[1] studied the vacuum solutions containing a congruence of diverging null geodesics, with vanishing shear and twist. The line element of these metrics can be expressed[4] by:

$$
d s^{2}=\left(-2 H r+K-2 \frac{M(u)}{r}\right) d u^{2}+2 d u d r-\frac{r^{2}}{P^{2}} d \zeta d \bar{\zeta}
$$

where $P=P(u, \zeta, \bar{\zeta}), H=\frac{\dot{P}}{P}, K=\Delta \ln P$, a doted quantity denotes its time derivative, a bar means complex conjugate and $\Delta$ is the two-dimensional Laplacian for the two-surfaces 
$u=$ constant, $r=$ constant with line element

$$
d S^{2}=\frac{1}{P^{2}} d \zeta d \bar{\zeta}
$$

where we are using complex stereographic coordinates $(\zeta, \bar{\zeta})$ for the sphere.

It is usually convenient to describe this line element in terms of the line element of the unit sphere; this is done by expressing $P$ as the product $P=V(u, \zeta, \bar{\zeta}) P_{0}(\zeta, \bar{\zeta})$, where $P_{0}$ is the value of $P$ for the unit sphere. If $\ell$ denotes the vector field that generates the null congruence, then $\ell=d u, \ell(r)=1, \ell(\zeta)=0$ and $\ell(\bar{\zeta})=0$. In other words this is the coordinate system adapted to the geometry. It is convenient to use the parameterization $u$ such that the mass parameter $M(u)=M_{0}=$ constant.

Alternatively one can express the geometry in terms of a null tetrad; since, given the complex null vector basis $\left(\ell^{a}, m^{a}, \bar{m}^{a}, n^{a}\right)$ with the properties:

$$
g_{a b} \ell^{a} n^{b}=-g_{a b} m^{a} \bar{m}^{b}=1
$$

and all other possible scalar products being zero, the metric can be expressed by

$$
g_{a b}=\ell_{a} n_{b}+n_{a} \ell_{b}-m_{a} \bar{m}_{b}-\bar{m}_{a} m_{b} .
$$

Using the coordinate system $\left(x^{0}, x^{1}, x^{2}, x^{3}\right)=\left(u, r,(\zeta+\bar{\zeta}), \frac{1}{i}(\zeta-\bar{\zeta})\right)$ defined above, one can express the null tetrad as:

$$
\begin{gathered}
\ell_{a}=(d u)_{a} \\
\ell^{a}=\left(\frac{\partial}{\partial r}\right)^{a} \\
m^{a}=\xi^{i}\left(\frac{\partial}{\partial x^{i}}\right)^{a} \\
\bar{m}^{a}=\bar{\xi}^{i}\left(\frac{\partial}{\partial x^{i}}\right)^{a} \\
n^{a}=U\left(\frac{\partial}{\partial r}\right)^{a}+X^{i}\left(\frac{\partial}{\partial x^{i}}\right)^{a}
\end{gathered}
$$

with $i=0,2,3$ and where the components $\xi^{i}, U$ and $X^{i}$ are:

$$
\xi^{0}=0, \quad \xi^{2}=\frac{\xi_{0}^{2}}{r}, \quad \xi^{3}=\frac{\xi_{0}^{3}}{r}
$$

with

$$
\xi_{0}^{2}=\sqrt{2} P_{0} V, \quad \xi_{0}^{3}=-i \xi_{0}^{2}
$$




$$
U=r U_{00}+U_{0}+\frac{U_{1}}{r}
$$

where

$$
U_{00}=\frac{\dot{V}}{V}, \quad U_{0}=-\frac{1}{2} K_{V}, \quad U_{1}=-\frac{\Psi_{2}^{0}+\bar{\Psi}_{2}^{0}}{2},
$$

where the curvature $K_{V}$ of the 2-metric appearing in equation (2), is given by

$$
K_{V}=\frac{2}{V} \bar{\jmath}_{V} \partial_{V} V-\frac{2}{V^{2}} ð_{V} V \bar{\jmath}_{V} V+V^{2},
$$

the leading order behavior $\Psi_{2}^{0}$ of the second component of the Weyl tensor is $\Psi_{2}^{0}=-M_{0}$ and

$$
X^{0}=1, \quad X^{2}=0, \quad X^{3}=0
$$

and where $\partial_{V}$ is the edth operator, in the GHP notation, of the sphere with metric (2).

In reference [1] it was found that the vacuum Einstein equation can be reduced to a parabolic equation for a scalar depending on three variables, the so called RobinsonTrautman equation; which in our notation has the form

$$
-3 M_{0} \dot{V}=V^{4} \check{\partial}^{2} \overline{\check{\partial}}^{2} V-V^{3} \check{\partial}^{2} V \bar{\partial}^{2} V
$$

where $\widetilde{\partial}$ is the edth operator of the unit sphere. We refer to a line element with $V$ satisfying this equation as a Robinson-Trautman solution. On the other hand, if the RT equation is not required, then the solution is no longer vacuum and there is only one component of the Ricci tensor different from zero, given by [5]

$$
\Phi_{22}^{(R T)}=\frac{-3 M_{0} \frac{\dot{V}}{V}-V^{3} \check{\partial}^{2} \overline{\mathrm{\partial}}^{2} V+V^{2} \check{\partial}^{2} V \overline{\mathrm{d}}^{2} V}{r^{2}}
$$

where the $(R T)$ is to emphasize the fact that in this case we are using the null tetrad adapted to the null congruence. We refer to this as a Robinson-Trautman geometry.

At this point it is interesting to remark that the spin coefficients $\sigma, \tau, \kappa, \sigma^{\prime}, \tau^{\prime}$ and $\epsilon$ are zero in the Robinson-Trautman geometries.

\section{Twisting algebraically special spacetimes}

\subsection{Null tetrad components of the exact solutions}

As it was indicated previously, from a geometrical point of view, the twisting algebraically special spacetimes are the natural generalizations of RT spacetimes, since they also have a congruence of null geodesics with vanishing shear and contain the RT metrics as the limit of those spaces when the twist goes to zero.

This kind of solutions have been extensively studied in the literature in terms of different tetrads and coordinate systems (for an account of these formulations of the 
problem see [8] and references therein). The tetrad and coordinate system used here do not agree with those ones. This new tetrad corresponds to what we call "standard rotating null tetrad"; they are defined in reference [16], and they can be applied to any asymptotically flat spacetime. In this section we will express the equations governing twisting algebraically special spacetimes in terms of the "standard rotating null tetrad".

The null tetrad components defined in equations (6) to (9) can be expressed by the three scalars $M(u, \zeta, \bar{\zeta}), V(u, \zeta, \bar{\zeta})$ and $L(u, \zeta, \bar{\zeta})$, and are given by:

$$
\xi^{0}=\frac{\xi_{0}^{0}}{r-i c}, \quad \xi^{2}=\frac{\xi_{0}^{2}}{r-i c}, \quad \xi^{3}=\frac{\xi_{0}^{3}}{r-i c},
$$

where

$$
\begin{gathered}
\xi_{0}^{0}=L V, \quad \xi_{0}^{2}=\sqrt{2} P_{0} V, \quad \xi_{0}^{3}=-i \xi_{0}^{2}, \quad P_{0}=\frac{1+\zeta \bar{\zeta}}{2} \\
U=r U_{00}+U_{0}+\frac{(M r+\mu c)}{(r+i c)(r-i c)}-\left(\frac{\tau_{0} \bar{\tau}_{00}}{r-i c}+\frac{\bar{\tau}_{0} \tau_{00}}{r+i c}\right)-\frac{\tau_{0} \bar{\tau}_{0}}{(r+i c)(r-i c)},
\end{gathered}
$$

where

$$
\begin{aligned}
& U_{00}=\frac{\dot{V}}{V}, \quad U_{0}=-\frac{1}{2}\left(K_{V}+K_{L}\right) \\
& K_{V}=2 V \bar{\partial} \check{\partial} V-2 \partial V \bar{\partial} V+V^{2}, \\
& K_{L}=2 \dot{\bar{L}} \dot{L} V^{2}+(\dot{L} \bar{L} V+\dot{\bar{L}} L V-2 ð V \bar{L}+V \precsim \bar{\partial}+V \bar{ð} L-2 \bar{ð} V L) \dot{V} \\
& +V^{2} \ddot{L} \bar{L}+2 \ddot{V} V L \bar{L}-2 \dot{V}^{2} L \bar{L}+V^{2} L \ddot{\bar{L}} \\
& +2 ð \dot{V} V(L+\bar{L})+V^{2}(\check{\partial} \bar{L}+\bar{\partial} L) \\
& \mu=\ddot{c} L V^{2} \bar{L}+2 \dot{c} \dot{L} V^{2} \bar{L}+\dot{\bar{c}} \dot{\bar{L}} L V^{2}+\dot{c} \bar{\partial} L V^{2} \\
& +\dot{c} c i+\frac{1}{2} \ddot{L} c V^{2} \bar{L}+\dot{L} \bar{\partial} c V^{2}+\frac{1}{2} \dot{L} \partial c L^{-1} V^{2} \bar{L}+\dot{L} c^{2} i L^{-1} \\
& +\frac{1}{2} \ddot{\bar{L}} c L V^{2}+\frac{1}{2} \dot{\bar{L}} \precsim c V^{2}+\bar{\jmath}(\dot{c}) L V^{2}+\frac{1}{2} \bar{\jmath} \dot{L} c V^{2}+\bar{\jmath} \text { ð } c V^{2} \\
& +\frac{1}{2} \bar{\partial} L ð c L^{-1} V^{2}+ð \dot{c} V^{2} \bar{L}+\frac{1}{2} ð \dot{\bar{L}} c V^{2} \\
& -\frac{1}{2} ð c ð \bar{L} L^{-1} V^{2}+c ð c i L^{-1}+c\left(K_{V}+K_{L}\right) \\
& \tau_{00}=-\dot{L} V, \\
& \tau_{0}=2 i c \tau_{00}-i V \partial_{L} c,
\end{aligned}
$$




$$
\begin{gathered}
c=-\frac{V^{2}}{2 i}\left(\bar{ळ}_{L} L-\widehat{\partial}_{L} \bar{L}\right) ; \\
X^{0}=1-\frac{\bar{\tau}_{00} \xi_{0}^{0}}{r-i c}-\frac{\tau_{00} \bar{\xi}_{0}^{0}}{r+i c}-\frac{\bar{\tau}_{0} \xi_{0}^{0}+\tau_{0} \bar{\xi}_{0}^{0}}{(r+i c)(r-i c)} \\
X^{2}=-\frac{\bar{\tau}_{00} \xi_{0}^{2}}{r-i c}-\frac{\tau_{00} \bar{\xi}_{0}^{2}}{r+i c}-\frac{\bar{\tau}_{0} \xi_{0}^{2}+\tau_{0} \bar{\xi}_{0}^{2}}{(r+i c)(r-i c)}, \\
X^{3}=-\frac{\bar{\tau}_{00} \xi_{0}^{3}}{r-i c}-\frac{\tau_{00} \bar{\xi}_{0}^{3}}{r+i c}-\frac{\bar{\tau}_{0} \xi_{0}^{3}+\tau_{0} \bar{\xi}_{0}^{3}}{(r+i c)(r-i c)}
\end{gathered}
$$

where the operator $\widetilde{\partial}_{L}$ acting on any function $f=f(u, \zeta, \bar{\zeta})$ is defined by:

$$
\mho_{L} f \equiv \precsim f+L \dot{f}
$$

and

$$
\overline{\widehat{\partial}}_{L} f \equiv \overline{\mathrm{\partial}} f+\bar{L} \dot{f}
$$

The spin coefficients corresponding to this tetrad are:

$$
\begin{gathered}
\rho=\frac{-1}{r+i c}, \\
\sigma=0, \\
\kappa=0, \\
\tau=\frac{\tau_{00}}{r+i c}+\frac{\tau_{0}}{(r+i c)(r-i c)}, \\
\rho_{0}^{\prime}=\frac{V \partial_{L} \tau_{0}^{\prime}-\bar{\partial}_{L} V \tau_{0}^{\prime}}{r-i c}+\frac{\Psi_{2}^{0} r}{(r+i c)^{2}(r+i c)}+\frac{i V \tau_{0}^{\prime} \partial_{L} c}{(r+i c)^{2}(r-i c)}, \\
\sigma^{\prime}=\frac{\sigma_{0}^{\prime}}{r+i c}-\frac{V \overline{\mathrm{\partial}}_{L} \tau_{0}^{\prime}+\overline{\bar{\partial}}_{L} V \tau_{0}^{\prime}}{(r+i c)^{2}}+\frac{i V \tau_{0}^{\prime} \overline{\mathrm{\partial}}_{L} c+\left(\tau_{0}^{\prime}\right)^{2}}{(r+i c)^{3}}, \\
\kappa^{\prime}=\frac{V \overline{\mathrm{d}}_{L} U}{r+i c}-U\left(\tau^{\prime}+\bar{\tau}\right),
\end{gathered}
$$




$$
\begin{gathered}
\tau^{\prime}=-\frac{\bar{\tau}_{0}}{(r+i c)^{2}}, \\
\epsilon=0, \\
\epsilon^{\prime}=\epsilon_{0}^{\prime}+\frac{\beta_{0} \bar{\tau}_{00}}{r-i c}-\frac{\bar{\beta}_{0} \tau_{00}}{r+i c}+\frac{\beta_{0} \bar{\tau}_{0}-\bar{\beta}_{0} \tau_{0}}{(r+i c)(r-i c)}+\frac{\Psi_{2}^{0}+2 \bar{\tau}_{0} \tau_{00}}{2(r+i c)^{2}}+\frac{\tau_{0} \bar{\tau}_{0}}{(r+i c)^{2}(r-i c)}, \\
\beta=\frac{\beta_{0}}{r-i c} \\
\beta^{\prime}=\bar{\beta}+\tau^{\prime} ;
\end{gathered}
$$

where

$$
\begin{gathered}
\rho_{0}^{\prime}=\frac{K_{V}+K_{L}}{2}-\dot{L} \dot{\bar{L}} V^{2}+\frac{\dot{V}}{V} i c-\frac{1}{2}\left(\bar{ळ}_{L} \dot{L}+\varlimsup_{L} \dot{\bar{L}}\right) V^{2}, \\
\sigma_{0}^{\prime}=-\dot{\bar{L}}^{2} V^{2}-\overline{\mathrm{\partial}}_{L}\left(\dot{\bar{L}} V^{2}\right) \\
\tau_{0}^{\prime}=-\bar{\tau}_{0} \\
\beta_{0}=-\frac{\sqrt{2} V\left(\frac{\partial P_{0}}{\partial x^{2}}-i \frac{\partial P_{0}}{\partial x^{3}}\right)+\partial_{L} V}{2} \\
\epsilon_{0}^{\prime}=\frac{\dot{V}}{2 V} .
\end{gathered}
$$

The Weyl tensor has the properties:

$$
\begin{gathered}
\Psi_{0}=0, \\
\Psi_{1}=0, \\
\Psi_{2}=\frac{\Psi_{2}^{0}}{(r+i c)^{3}},
\end{gathered}
$$

where

$$
\Psi_{2}^{0}=-(M+i \mu)
$$

The components $\Psi_{3}$ and $\Psi_{4}$ are too complicated to be presented here. 
The vacuum equations require several relations among the main functions. The equation $\Phi_{12}=0$ imposes the condition:

$$
\partial_{L}(M+i \mu)=-3 \dot{L}(M+i \mu) .
$$

The other relation has a compact form if one uses the function $z(u, \zeta, \bar{\zeta})$ defined by

$$
V=\frac{\partial z}{\partial u}=\dot{z} ;
$$

using this, the equation $\Phi_{22}=0$ imposes the relation

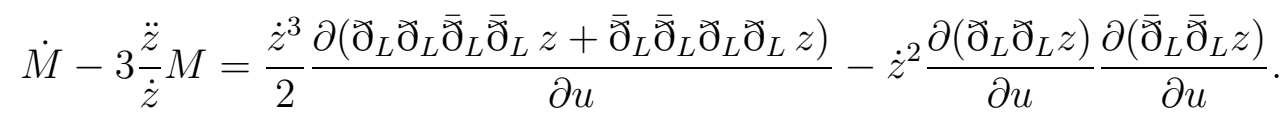

It is interesting to note that using $z$ the quantity $\mu$ acquires the compact form

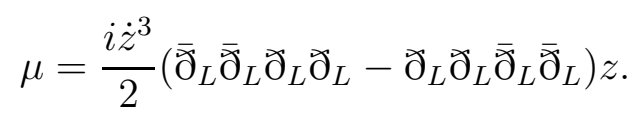

It is easy to see that this tetrad goes to the RT tetrad when $L$ goes to zero.

The representation of the twisting algebraically special spacetimes in terms of standard rotating null tetrads, we have just gave, is different from that appearing in the book of exact solutions by Kramer et.al. [8] and its references, because we use a different tetrad frame.

The twist of the null congruence, and also the angular momentum of the spacetime, are proportional to the quantity $c$ defined above.

The complex equation (54) and the real equation (56) constitute three equations for the real functions $z$ and $M$ and the complex function $L$; in other words one has three real

equations for four real scalars. In order to understand this under determined system it is important to mention the gauge freedom available.

\subsection{The gauge freedom}

These solutions are asymptotically flat. The retarded time null coordinate $u$ defines, at future null infinity, a set of sections by the equations $u=$ constant. The main gauge freedom can be associated to a different choice of these sections; therefore let us consider a transformation of the form

$$
\begin{gathered}
\tilde{u}=\tilde{u}_{0}(u, \zeta, \bar{\zeta})+O\left(\frac{1}{r}\right), \\
\tilde{r}=\frac{r}{w(u, \zeta, \bar{\zeta})}+r_{0}(u, \zeta, \bar{\zeta}), \\
\tilde{\zeta}=\zeta+O\left(\frac{1}{r}\right) ;
\end{gathered}
$$


one could also generalized the last equation to a transformation among the sphere coordinates $(\zeta, \bar{\zeta})$, but they are not important for the following equations and therefore we do not consider them main gauge freedom.

The null character of the coordinate $\tilde{u}$ requires

$$
w=\dot{\tilde{u}}_{0}
$$

Although the vector $\tilde{\ell}$ is proportional to $\ell$, the other tetrad vectors rotate under this transformation. From the study of the rotations one deduces that

$$
\begin{gathered}
\tilde{V}=\frac{V}{w}, \\
\tilde{V}\left(\tilde{L}-\widetilde{\partial} \tilde{u}_{0}\right)=V L
\end{gathered}
$$

and

$$
\tilde{M}+i \tilde{\mu}=\frac{M+i \mu}{w^{3}}
$$

Let us observe that there are several possibilities associated to the three equations (62), (63) and (64). If the twist of the null congruence is different from zero then one can choose $u_{0}(u, \zeta, \bar{\zeta})$ so that $\tilde{V}=1$, for example.

If we write the spin weight 1 quantity $L$ in terms of the spin weight 0 quantity $J$ by the relation

$$
L=i ð J=i ð \Re e J-ð \Im m J
$$

where the complex scalar $J$ can be expressed in term of its real and imaginary parts $\Re e J$ and $\Im m J$, so that $J=\Re e J+i \Im m J$; then equation (63) can be written in the form

$$
i ð_{\tilde{V}} \tilde{J}-\mho_{\tilde{V}} \tilde{u}_{0}=i ð_{V} J
$$

where $\partial_{\tilde{V}}$ is the edth operator for the sphere with metric $d S^{2}=\frac{1}{\tilde{V}^{2} P_{0}^{2}} d \zeta d \bar{\zeta}$. From this equation it can be seen that in principle one could use $u_{0}$ to make $\Im m \tilde{J}$ vanish; but it is not clear that the needed $u_{0}$ would satisfy that $\dot{u}_{0}>0$; as it is required for a non-singular transformation. If $\Im m \dot{J}$ where zero then one can see that a transformation of the form $\tilde{u}=u-\gamma(\zeta, \bar{\zeta})$ would do the job. It is conceivable that for small time variations one can use techniques similar to those of references [11] and [14 to prove local existence of transformations that make $\Im m \tilde{J}=0$.

Another possibility is to use the freedom involved in the choice of $u_{0}$ to make $\tilde{M}$ to be a constant. This can always be made since from equation (64) one always solve for $\tilde{u}_{0}$, since the function $M$, for physically realistic spacetimes, is assumed to be positive definite. This choice shows that from the four original unspecified real scalars, one of them is pure gauge; therefore the complex equation (54) and the real equation (56) constitute a system of three equations for three physical real scalars. 


\subsection{Algebraically special perturbations of RT spacetimes}

Having just presented the exact algebraically special solutions in terms of a new frame, we proceed here with our program by calculating the equations governing the algebraically special perturbations of RT spacetimes. We will also specialize the discussion to the asymptotic future regime for the retarded time going to infinity, where the equations are solved.

When one studies the case of a linear perturbation of the RT line element, one considers:

$$
V=V_{R T}+V_{L}
$$

and

$$
M=M_{0}+M_{L}
$$

where $V_{R T}$ satisfies the RT equation, $M_{0}$ is the constant RT mass and $V_{L}$ and $M_{L}$ represents the departure from the RT metric that take into account the presence of angular momentum; which are zero when $L$ vanishes.

From equation $\Phi_{12}=0$ it is obtained, in first order

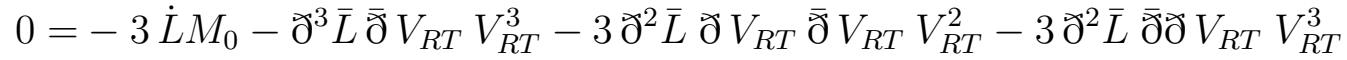

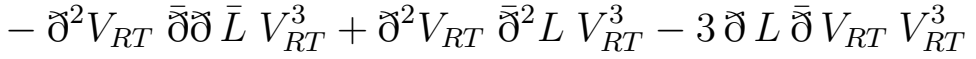

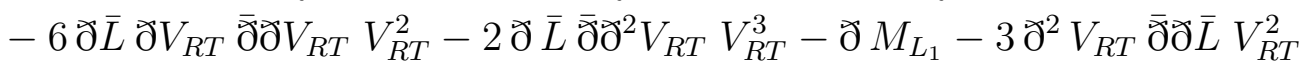

$$
\begin{aligned}
& +3 \check{\partial}^{2} V_{R T} \overline{\mathrm{\partial}}^{2} L V_{R T}^{2}-3 \partial V_{R T} \overline{\mathrm{\partial}} \varlimsup^{2} \bar{L} V_{R T}^{3}+3 \check{\partial} V_{R T} \overline{\mathrm{\partial}} \mathrm{\partial} L \overline{\mathrm{\partial}} V_{R T} V_{R T}^{2}
\end{aligned}
$$

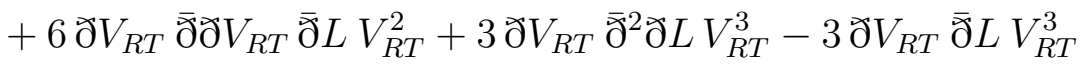

$$
\begin{aligned}
& -3 ð V_{R T} \bar{\partial} V_{R T} L V_{R T}^{2}-\frac{1}{2} \overline{\bar{\partial}} \varlimsup^{3} \bar{L} V_{R T}^{4}+\bar{\varnothing} \check{\partial}^{2} L \bar{\partial} V_{R T} V_{R T}^{3}
\end{aligned}
$$

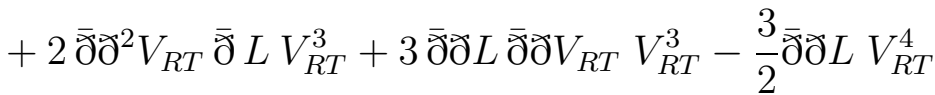

$$
\begin{aligned}
& -3 \overline{\widetilde{\partial}} V_{R T} L V_{R T}^{3}+\frac{1}{2} \overline{\check{\partial}}^{2} \check{\partial}^{2} L V_{R T}^{4}
\end{aligned}
$$


while from the equation $\Phi_{22}=0$ the following relation is obtained

$$
\begin{aligned}
& 0=-2 \dot{L} \partial V_{R T} \bar{\partial}^{2} V_{R T} V_{R T}^{3}+2 \dot{L} \bar{\partial}^{2} ð V_{R T} V_{R T}^{4}+\dot{L} \bar{\partial} V_{R T} V_{R T}^{4}-2 \dot{\bar{L}} \check{\partial}^{2} V_{R T} \bar{\partial} V_{R T} V_{R T}^{3} \\
& +2 \dot{\bar{L}} \bar{\partial} \check{\partial}^{2} V_{R T} V_{R T}^{4}-\dot{M}_{L} V_{R T}+3 \dot{V}_{L} M_{0}-\dot{V}_{R T} \check{\partial}^{2} V_{R T} \bar{\jmath} \bar{L} V_{R T}^{3}
\end{aligned}
$$

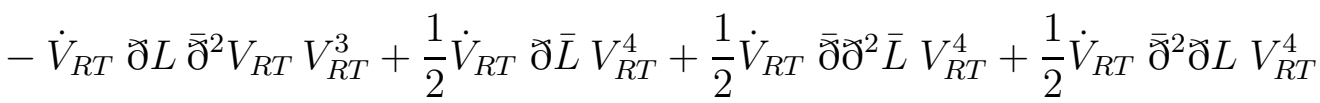

$$
\begin{aligned}
& -12 \frac{\dot{V}_{R T}}{V_{R T}} M_{0} V_{L}+3 \dot{V}_{R T} M_{L}+2 \precsim \dot{\bar{L}} \overline{\mathrm{\partial}} \mathrm{\partial} V_{R T} V_{R T}^{4}+\frac{1}{2} ð \dot{\bar{L}} V_{R T}^{5}+ð \dot{V}_{R T} \bar{\jmath} \precsim \bar{\partial} V_{R T}^{4} \\
& +\precsim \dot{V}_{R T} \bar{\partial}^{2} L V_{R T}^{4}-2 ð \dot{V}_{R T} \bar{\partial}^{2} V_{R T} L V_{R T}^{3}+\check{\partial}^{2} \dot{\bar{L}} \overline{\mathrm{\partial}} V_{R T} V_{R T}^{4}+\varlimsup^{2} \dot{V}_{R T} \overline{\mathrm{\partial}} \bar{L} V_{R T}^{4} \\
& +\check{\partial}^{2} \bar{L} \bar{\partial} \dot{V}_{R T} V_{R T}^{4}-\check{\partial}^{2} V_{L} \overline{\check{\partial}}^{2} V_{R T} V_{R T}^{3}-2 \check{\partial}^{2} V_{R T} \bar{\partial} \dot{V}_{R T} \bar{L} V_{R T}^{3} \\
& -\check{\partial}^{2} V_{R T} \overline{\check{\partial}}^{2} V_{L} V_{R T}^{3}+\check{\partial}^{2} V_{R T} \overline{\check{\partial}}^{2} V_{R T} V_{L} V_{R T}^{2}+ð L \bar{\partial}^{2} \dot{V}_{R T} V_{R T}^{4}
\end{aligned}
$$

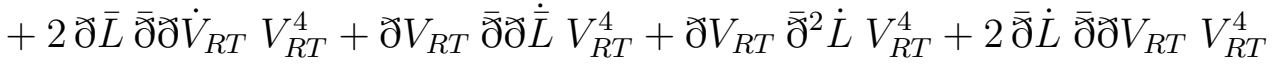

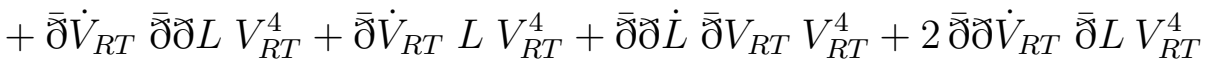

$$
\begin{aligned}
& +\frac{1}{2} \overline{\bar{\partial}} \check{\partial}^{2} \dot{\bar{L}} V_{R T}^{5}+2 \bar{\jmath}^{2} \dot{V}_{R T} \bar{L} V_{R T}^{4}+\frac{1}{2} \bar{\partial}^{2} ð \dot{L} V_{R T}^{5}+2 \overline{\check{\partial}}^{2} \check{\partial} \dot{V}_{R T} L V_{R T}^{4} \\
& +\overline{\widetilde{\partial}}^{2} \check{\partial}^{2} V_{L} V_{R T}^{4}
\end{aligned}
$$

In the asymptotic future; for $u \rightarrow \infty$ it is known 6 that

$$
V_{R T}=1+O\left(e^{-\frac{2 u}{M_{0}}}\right)
$$

therefore to first order in the asymptotic expansion of the retarded time, the above two equations become

$$
0=-3 \dot{L}_{1} M_{0}-\text { ð } M_{L_{1}}-\frac{1}{2} \bar{ð} \varlimsup^{3} \bar{L}_{1}-\frac{3}{2} \bar{ð} ð L_{1}+\frac{1}{2} \bar{ð}^{2} \check{\partial}^{2} L_{1}
$$

and

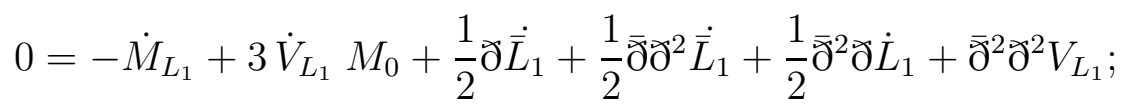

where the subindex 1 means that we are considering first order quantities in the expansion as $u \rightarrow \infty$.

Using the complex scalar $J$ which satisfies (65), equations (72) and (73) become

$$
0=3 ð\left(\Im m \dot{J}_{1}\right) i M_{0}+3 ð\left(\Re e \dot{J}_{1}\right) M_{0}-ð M_{L_{1}} i-ð\left(\bar{\partial}^{2} \check{\partial}^{2} \Re e J_{1}\right)
$$

and

$$
0=-\dot{M}_{L_{1}}+3 \dot{V}_{L_{1}} M_{0}-\bar{\varlimsup}^{2} \check{\partial}^{2} \Im m \dot{J}_{1}+\bar{\jmath}^{2} \check{\partial}^{2} V_{L_{1}}
$$

where it can be seen from equation (74) that if we use the gauge freedom to make $M_{L_{1}}$ a constant, then $\precsim \Im m \dot{J}_{1}$ must vanish which implies that $\Im m \dot{J}_{1}=0$. It is clear from the 
discussion of the previous subsection that by means of a further gauge transformation one can make $\Im m J_{1}=0$. Therefore in this gauge the above equations become

$$
0=ð\left(3 \dot{J}_{1} M_{0}-\bar{\partial}^{2} \check{\partial}^{2} J_{1}\right)
$$

where now $J_{1}$ is assumed to be real, and

$$
0=3 \dot{V}_{L_{1}} M_{0}+\overline{\mathrm{\partial}}^{2} \check{\partial}^{2} V_{L_{1}}
$$

The constant $M_{L_{1}}$ now plays no role, and it can be made to vanish by a further reparameterization of the retarded time coordinate. Integrating equation (76) we can write these two equations in a more comparable form

$$
3 \dot{J}_{1} M_{0}=\overline{\mathrm{\partial}}^{2} \check{\partial}^{2} J_{1}
$$

and

$$
-3 \dot{V}_{L_{1}} M_{0}=\overline{\widetilde{\partial}}^{2} \check{\partial}^{2} V_{L_{1}}
$$

It is important to know the spectrum of the operator appearing on the right hand side. The eigenvalues of the operator $\bar{\partial}^{2} \partial^{2}$ are 11] $\frac{(\ell-1) \ell(\ell+1)(\ell+2)}{4}$; where $\ell$ is any non-negative integer.

Therefore the leading order behavior for the functions are:

$$
J_{1}=J_{1}^{m} Y_{1 m}(\zeta, \bar{\zeta})+J_{2}^{m} Y_{2 m}(\zeta, \bar{\zeta}) e^{\frac{2 u}{M_{0}}}+O\left(e^{\frac{4 u}{M_{0}}}\right)
$$

and

$$
V_{L_{1}}=V_{L_{1}}^{m} Y_{2 m}(\zeta, \bar{\zeta}) e^{-\frac{2 u}{M_{0}}}+O\left(e^{-\frac{4 u}{M_{0}}}\right)
$$

where $J_{1}^{m}, J_{2}^{m}$ and $V_{L_{1}}^{m}$ are constants and we have used gauge transformations to set to zero spurious integrations constants.

It is observed that $V_{L_{1}}$ has the same asymptotic behavior as $V_{R T}$; while $J_{1}$ has a constant angular momentum term and a possible divergent behavior for the higher moments in the limit $u \rightarrow \infty$.

Therefore these spacetimes are not suitable for the description of a perturbed black hole with a dynamic angular momentum. This forces us to consider general perturbations of RT spacetimes, that we treat in the next section.

\section{General perturbations of RT spacetimes}

\subsection{The vacuum equations}

Let us consider now a general perturbation of the RT spacetimes. We will still work with a null tetrad satisfying equations (3-9), with $\xi^{0}=0$, and the leading order of $\xi^{2}$ and $\xi^{3}$ also given by eq. (11); but where now the scalar $V$ is given by

$$
V=V_{R T}+\lambda V_{\lambda}
$$


where $V_{R T}$ is the RT scalar discussed in section 2, $\lambda$ the linearization parameter and $V_{\lambda}$ the linear perturbation scalar. Let us emphasize that this means that the tetrad vector $\ell^{a}$ has no twist, unlike the corresponding one in the rotating null tetrad used in the last section. In this section we use asymptotic coordinates and standard tetrad as defined in [16]; however it is important to notice that we do not use a Bondi system. We use $\lambda$ to emphasize in some equations the linearized terms; but it should be understood that it has the value 1 at any stage.

It is convenient to start the study from the vacuum Bianchi identities.

Assume that in the null hypersurface $\Sigma$, defined by $u=u_{0}$, of the original RT spacetime one is given the function $\Psi_{0}\left(u_{0}, r, \zeta, \bar{\zeta}\right)$. Then, noting that $\Psi_{0}$ and $\Psi_{1}$ are zero in the Robinson-Trautman geometries; one obtains, from eq. (3.91) in ref. [10] that, on $\Sigma, \Psi_{1}$ is given by

$$
\Psi_{1}=\frac{\Psi_{1}^{0}}{r^{4}}+\frac{1}{r^{4}} \int^{r} r^{\prime 3} \overline{\widehat{\partial}}_{V_{R T}} \Psi_{0} d r^{\prime}
$$

where $\Psi_{1}^{0}=\Psi_{1}^{0}\left(u_{0}, \zeta, \bar{\zeta}\right)$.

Similarly, from eq, (3.90) of ref. [10] one obtains that $\Psi_{2}$ is given on $\Sigma$ by

$$
\Psi_{2}=\frac{\Psi_{2}^{0}}{r^{3}}+\frac{1}{r^{3}} \int^{r} r^{\prime 2} \overline{\bar{\partial}}_{V_{R T}} \Psi_{1} d r^{\prime}
$$

with

$$
\Psi_{2}^{0}=-\left(M_{0}+\lambda\left(M_{1}+i \mu\right)\right),
$$

where $M_{0}$ is the constant RT mass, $M_{1}=M_{1}\left(u_{0}, \zeta, \bar{\zeta}\right)$ and $\mu=\mu\left(u_{0}, \zeta, \bar{\zeta}\right)$ are real functions and $\partial_{V_{R T}}$ is the edth operator of the 2-sphere with the RT conformal factor.

When calculating $\Psi_{3}$ from eq. (3.89) of ref. [10], one should take into account the first order contribution coming from the edth primed operator; due to the fact that $\Psi_{2}$ has zero order terms.

In order to proceed, we need to calculate the first order perturbation of the spin coefficients $\rho$ and $\sigma$.

Equation (3.50) of [10] is

$$
\frac{\partial \rho}{\partial r}=\rho^{2}
$$

which has solution

$$
\rho=-\frac{1}{r}
$$

after appropriately chosen the freedom in the origin of the coordinate $r$.

Then eq. (3.51) of 10 becomes

$$
\frac{\partial \sigma}{\partial r}=2 \rho \sigma+\Psi_{0}
$$


which has solution

$$
\sigma=\frac{\sigma_{0}}{r^{2}}+\frac{1}{r^{2}} \int^{r} r^{\prime 2} \Psi_{0} d r^{\prime}
$$

where $\sigma_{0}=\sigma_{0}\left(u_{0}, \zeta, \bar{\zeta}\right)$.

From eq. (3.52) of [10] one obtains

$$
\frac{\partial \tau}{\partial r}=2 \rho \tau+\Psi_{1}
$$

with solution

$$
\tau=\frac{\tau_{0}}{r^{2}}+\frac{1}{r^{2}} \int^{r} r^{\prime 2} \Psi_{1} d r^{\prime} ;
$$

where $\tau_{0}=\tau_{0}\left(u_{0}, \zeta, \bar{\zeta}\right)$.

One can now integrate eq. (3.31) of [10] to obtain the radial dependence of $\xi^{i}$. It is deduced that

$$
\xi^{i}=\frac{\xi_{0}^{i}}{r}+\frac{1}{r} \int^{r} r^{\prime} \sigma \bar{\xi}^{i} d r^{\prime}
$$

where $\xi_{0}^{i}$ does not depend on $r$; then, one can write the integral equation

$$
\xi^{i}=\frac{\xi_{0}^{i}}{r}+\frac{1}{r} \int^{r} r^{\prime} \sigma\left(\frac{\bar{\xi}_{0}^{i}}{r^{\prime}}+\frac{1}{r^{\prime}} \int^{r^{\prime}} r^{\prime \prime} \bar{\sigma} \xi^{i} d r^{\prime \prime}\right) d r^{\prime} ;
$$

which can be solved by iterations. But, since we should consider only linear terms on $\lambda$, one obtains that in first order

$$
\begin{aligned}
\xi^{i} & =\frac{\xi_{0}^{i}}{r}+\frac{1}{r} \int^{r} \sigma \bar{\xi}_{0}^{i} d r^{\prime} \\
& =\frac{\xi_{0}^{i}}{r}-\frac{\bar{\xi}_{0}^{i} \sigma_{0}}{r^{2}}+\frac{\bar{\xi}_{0}^{i}}{r} \int^{r}\left(\frac{1}{r^{\prime 2}} \int^{r^{\prime}} r^{\prime \prime 2} \Psi_{0} d r^{\prime \prime}\right) d r^{\prime}
\end{aligned}
$$

The radial dependence of the components $X^{i}$ is obtained from eq. (3.28) of [10]; which is

$$
\frac{\partial X^{i}}{\partial r}=2 \bar{\tau} \xi^{i}+2 \tau \bar{\xi}^{i}
$$

which implies, in first order, that

$$
X^{i}=X_{0}^{i}+\left[2 \bar{\xi}_{0}^{i} \int^{r}\left(\frac{\tau_{0}}{r^{\prime 3}}+\frac{1}{r^{\prime 3}} \int^{r^{\prime}} r^{\prime \prime 2} \Psi_{1} d r^{\prime \prime}\right) d r^{\prime}+\text { c.c. }\right]
$$

where c.c. means complex conjugate and the $X_{0}^{i}$ do not depend on $r$; but from the coordinate condition at future null infinity[16], one actually has $X_{0}^{i}=0$. 
In order to determine the dependence of $U$ on the coordinate $r$, one could use eq. (3.26) of [10]; which requires to calculate $\epsilon^{\prime}$ first. Equation (3.58) of the same reference is in our case

$$
\frac{\partial \epsilon^{\prime}}{\partial r}=-2 \bar{\tau} \beta+2 \tau \beta^{\prime}-\Psi_{2}
$$

therefore

$$
\begin{aligned}
\frac{\partial\left(\epsilon^{\prime}+\bar{\epsilon}^{\prime}\right)}{\partial r} & =-2 \bar{\tau}\left(\beta-\bar{\beta}^{\prime}\right)+2 \tau\left(\beta^{\prime}-\bar{\beta}\right)-\left(\Psi_{2}+\bar{\Psi}_{2}\right) \\
& =-4 \tau \bar{\tau}-\left(\Psi_{2}+\bar{\Psi}_{2}\right)
\end{aligned}
$$

where we must neglect the product involving $\tau$; since it is a quantity of higher order. One concludes that

$$
\frac{\partial^{2} U}{\partial r^{2}}=-\left(\Psi_{2}+\bar{\Psi}_{2}\right)
$$

which implies that $U$ is of the form

$$
U=r U_{00}+U_{0}-\int^{r} \int^{r^{\prime}}\left(\Psi_{2}+\bar{\Psi}_{2}\right) d r^{\prime \prime} d r^{\prime} ;
$$

where $U_{00}$ and $U_{0}$ do not depend on $r$.

The $r$ dependence of the tetrad components can be explicitly given in terms of the potential $W_{0}(u, r, \zeta, \bar{\zeta})$, defined from

$$
\Psi_{0}=\frac{\partial^{4} W_{0}}{\partial r^{4}}
$$

by the relations

$$
\begin{aligned}
& \xi^{0}=0 \\
& \xi^{2}=\frac{\xi_{0}^{2}}{r}+\lambda \bar{\xi}_{0}^{2}\left(-\frac{\sigma_{0}}{r^{2}}+\frac{1}{r} \frac{\partial^{2} W_{0}}{\partial r^{2}}-\frac{2}{r^{2}} \frac{\partial W_{0}}{\partial r}\right), \\
& \xi^{3}=\frac{\xi_{0}^{3}}{r}+\lambda \bar{\xi}_{0}^{3}\left(-\frac{\sigma_{0}}{r^{2}}+\frac{1}{r} \frac{\partial^{2} W_{0}}{\partial r^{2}}-\frac{2}{r^{2}} \frac{\partial W_{0}}{\partial r}\right)
\end{aligned}
$$

with

$$
\begin{gathered}
\xi_{0}^{2}=\sqrt{2} P_{0} V, \quad \xi_{0}^{3}=-i \xi_{0}^{2} \\
U=r U_{00}+U_{0}+\frac{U_{1}}{r}+\frac{U_{2}}{r^{2}}+\Delta U_{3},
\end{gathered}
$$


where

$$
\begin{aligned}
U_{00} & =\frac{\dot{V}}{V} \\
U_{0} & =-\frac{1}{2} K_{V}, \\
U_{1} & =-\frac{1}{2}\left(\Psi_{2}^{0}+\bar{\Psi}_{2}^{0}\right), \\
U_{2} & =\frac{\lambda}{6}\left(\check{\partial}_{V_{R T}} \bar{\Psi}_{1}^{0}+\overline{\mathrm{\partial}}_{V_{R T}} \Psi_{1}^{0}\right), \\
\Delta U_{3} & =-\frac{\lambda}{r^{2}}\left(\overline{\widetilde{\partial}}_{V_{R T}}^{2} W_{0}+\check{\partial}_{V_{R T}}^{2} \bar{W}_{0}\right) ;
\end{aligned}
$$

and

$$
\begin{aligned}
& X^{0}=1 \text {, } \\
& X^{2}=\lambda \xi_{0}^{2}\left(-\frac{\bar{\tau}_{0}}{r^{2}}+\frac{2 \bar{\Psi}_{1}^{0}}{3 r^{3}}+\frac{2}{r^{2}} \frac{\partial \varlimsup_{V_{R T}} \bar{W}_{0}}{\partial r}-\frac{4}{r^{3}}{{ }_{V_{R T}}}_{W_{0}}\right)+\text { c.c. }, \\
& X^{3}=\lambda \xi_{0}^{3}\left(-\frac{\bar{\tau}_{0}}{r^{2}}+\frac{2 \bar{\Psi}_{1}^{0}}{3 r^{3}}+\frac{2}{r^{2}} \frac{\partial \oiint_{V_{R T}} \bar{W}_{0}}{\partial r}-\frac{4}{r^{3}} \partial_{V_{R T}} \bar{W}_{0}\right)+\text { c.c. } ;
\end{aligned}
$$

where

$$
\begin{gathered}
\tau_{0}=\overline{\mathrm{\partial}}_{V_{R T}} \sigma_{0}, \\
K_{V}=\frac{2}{V} \bar{\partial}_{V} \varlimsup_{V} V-\frac{2}{V^{2}} \partial_{V} V \bar{\partial}_{V} V+V^{2} .
\end{gathered}
$$

In these equations we are explicitly denoting the first order terms by introducing the $\lambda$ dependency. The scalar $V$ is given by equation (82), and therefore one can express $K_{V}$ by

$$
K_{V}=K_{V_{R T}}+\lambda K_{V_{\lambda}}
$$

where

$$
K_{V_{R T}}=\frac{2}{V_{R T}} \overline{\mathrm{\partial}}_{V_{R T}} \partial_{V_{R T}} V_{R T}-\frac{2}{V_{R T}^{2}} \partial_{V_{R T}} V_{R T} \overline{\bar{\partial}}_{V_{R T}} V_{R T}+V_{R T}^{2}
$$

and

$$
\begin{aligned}
& K_{V_{\lambda}}=\frac{2}{V_{R T}} \overline{\bar{\partial}}_{V_{R T}} \partial_{V_{R T}} V_{\lambda}-\frac{2}{V_{R T}^{2}} \partial_{V_{R T}} V_{\lambda} \bar{\partial}_{V_{R T}} V_{R T}-\frac{2}{V_{R T}^{2}} \partial_{V_{R T}} V_{R T} \overline{\bar{\partial}}_{V_{R T}} V_{\lambda} \\
& +\frac{2 V_{\lambda}}{V_{R T}^{3}} \varlimsup_{V_{R T}} V_{R T} \overline{\mathrm{\partial}}_{V_{R T}} V_{R T}+V_{\lambda} V_{R T}+\frac{V_{\lambda}}{V_{R T}} K_{V_{R T}} .
\end{aligned}
$$

The previous relations make the following spinor components of the Ricci tensor to vanish

$$
\Phi_{00}=0,
$$




$$
\begin{aligned}
& \Phi_{01}=0, \\
& \Phi_{11}=0
\end{aligned}
$$

and

$$
\Lambda=0
$$

The other equations, coming from the other spinor components, involve time derivatives of the functions. They are:

$$
\begin{aligned}
& \frac{\partial^{2}\left(\frac{W_{0}}{r^{3}}\right)}{\partial u \partial r}=-\frac{1}{r^{2}} \frac{\dot{V}_{R T}}{V_{R T}} \frac{\partial^{2} W_{0}}{\partial r^{2}}+\frac{1}{r^{3}}\left(4 \frac{\dot{V}_{R T}}{V_{R T}} \frac{\partial W_{0}}{\partial r}+\frac{1}{2} K_{V_{R T}} \frac{\partial^{2} W_{0}}{\partial r^{2}}\right) \frac{1}{r^{4}}\left(-6 \frac{\dot{V}_{R T}}{V_{R T}} W_{0}-K_{V_{R T}} \frac{\partial W_{0}}{\partial r}-M_{0} \frac{\partial^{2} W_{0}}{\partial r^{2}}\right) \\
&+\frac{1}{r^{5}}\left(-\frac{1}{6} \partial_{V_{R T}} \Psi_{1}^{0}+\bar{\jmath}_{V_{R T}} \partial_{V_{R T}} W_{0}+M_{0} \frac{\partial W_{0}}{\partial r}+\frac{1}{2} M_{0} \sigma_{0}-2 K_{V_{R T}} W_{0}\right), \\
& \dot{\Psi}_{1}^{0}=3 \frac{\dot{V}_{R T}}{V_{R T}} \Psi_{1}^{0}-\varlimsup_{V_{R T}}\left(M_{1}+i \mu\right)+\bar{\varpi}_{V_{R T}}\left(K_{V_{R T}}\right) \sigma_{0},
\end{aligned}
$$

where $\mu$ is actually related to $\sigma_{0}$ by

$$
\mu=\frac{1}{2 i}\left(\check{\partial}_{V_{R T}}^{2} \bar{\sigma}_{0}-\overline{\mathrm{\partial}}_{V_{R T}}^{2} \sigma_{0}\right)
$$

the zero order term of $\Phi_{22}=0$ gives

$$
-6 M_{0} \frac{\dot{V}_{R T}}{V_{R T}}=\overline{\mathrm{\partial}}_{V_{R T}} \mathrm{\partial}_{V_{R T}} K_{V_{R T}},
$$

which is just another way to write the RT equation, and from the first order one obtains

$$
\begin{aligned}
& -6 M_{0} \frac{\dot{V}_{\lambda}}{V_{R T}}=\overline{\mathrm{\partial}}_{V_{R T}} \varlimsup_{V_{R T}} K_{V_{\lambda}}-6 \frac{\dot{V}_{R T}}{V_{R T}}\left(3 M \frac{V_{\lambda}}{V_{R T}}-M_{1}\right)-2 \dot{M}_{1}
\end{aligned}
$$

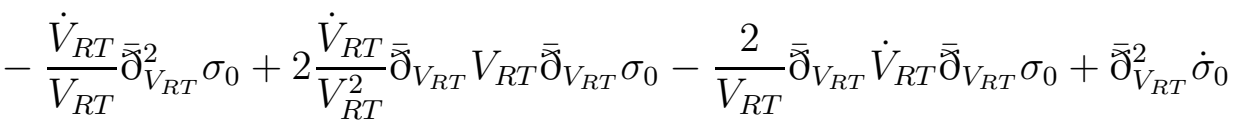

$$
\begin{aligned}
& -\frac{\dot{V}_{R T}}{V_{R T}} \varlimsup_{V_{R T}}^{2} \bar{\sigma}_{0}+2 \frac{\dot{V}_{R T}}{V_{R T}^{2}} \varlimsup_{V_{R T}} V_{R T} \varlimsup_{V_{R T}} \bar{\sigma}_{0}-\frac{2}{V_{R T}} ð_{V_{R T}} \dot{V}_{R T} ð_{V_{R T}} \bar{\sigma}_{0}+\varlimsup_{V_{R T}}^{2} \dot{\bar{\sigma}}_{0} .
\end{aligned}
$$

One could also consider the leading order asymptotic expansion of $W_{0}$ by writing

$$
W_{0}=\frac{\Psi_{0}^{0}}{4 ! r}+W_{1}
$$


where $\Psi_{0}^{0}=\Psi_{0}^{0}(u, \zeta . \bar{\zeta})$; in this way one has

$$
\Psi_{0}=\frac{\Psi_{0}^{0}}{r^{5}}+\frac{\partial^{4} W_{1}}{\partial r^{4}}
$$

where the second term is of order $O\left(1 / r^{6}\right)$.

In this case equation (116) splits into two equations, namely

$$
\dot{\Psi}_{0}^{0}=3 \frac{\dot{V}_{R T}}{V_{R T}} \Psi_{0}^{0}+{\stackrel{\partial}{V_{R T}}}_{1} \Psi_{1}^{0}-3 M_{0} \sigma_{0}
$$

and

$$
\begin{aligned}
\frac{\partial^{2}\left(\frac{W_{1}}{r^{3}}\right)}{\partial u \partial r}=-\frac{1}{r^{2}} \frac{\dot{V}_{R T}}{V_{R T}} & \frac{\partial^{2} W_{1}}{\partial r^{2}}+\frac{1}{r^{3}}\left(4 \frac{\dot{V}_{R T}}{V_{R T}} \frac{\partial W_{1}}{\partial r}+\frac{1}{2} K_{V_{R T}} \frac{\partial^{2} W_{1}}{\partial r^{2}}\right) \\
& +\frac{1}{r^{4}}\left(-6 \frac{\dot{V}_{R T}}{V_{R T}} W_{1}-K_{V_{R T}} \frac{\partial W_{1}}{\partial r}-M_{0} \frac{\partial^{2} W_{1}}{\partial r^{2}}\right) \\
+ & \frac{1}{r^{5}}\left(+\overline{\bar{\partial}}_{V_{R T}} \partial_{V_{R T}} W_{1}+M_{0} \frac{\partial W_{1}}{\partial r}+\frac{1}{2} M_{0} \sigma_{0}-2 K_{V_{R T}} W_{1}\right) \\
& +\frac{1}{r^{6}}\left(+\frac{1}{24} \overline{\bar{\partial}}_{V_{R T}} \partial_{V_{R T}} \Psi_{0}^{0}\right)-\frac{1}{8 r^{7}} \Psi_{0}^{0} M_{0} .
\end{aligned}
$$

Let us notice that equations (124), (123), (117) and (120) are the evolution equations for the first order quantities $W_{1}, \Psi_{0}^{0}, \Psi_{1}^{0}$ and $V_{\lambda}$ and $M_{1}$ respectively; while equation (119) (the RT equation) is the evolution equation for the zero order quantity $V_{R T}$. The scalar $\sigma_{0}$ is an arbitrary complex function that affects the radiation content of the spacetime. To understand these equations it is important to study the gauge freedoms considered in the next subsection.

\subsection{The gauge freedom}

The main gauge freedom admitted in our calculation is of the form

$$
\begin{aligned}
\tilde{u} & =\tilde{u}_{0}(u, \zeta, \bar{\zeta})+\frac{\tilde{u}_{1}(u, \zeta, \bar{\zeta})}{r}+O\left(\frac{1}{r^{2}}\right), \\
\tilde{r} & =\frac{r}{w(u, \zeta, \bar{\zeta})}+O\left(r^{0}\right) \\
\tilde{\zeta} & =\zeta+O\left(\frac{1}{r}\right)
\end{aligned}
$$

one could also admit a further transformation of the coordinates of the sphere $(\zeta, \bar{\zeta})$ into itself, that was also available in the original Robinson-Trautman geometry; but this will not change the following result.

The condition $g^{\tilde{u} \tilde{r}}=1$ imposes the relation

$$
w=\dot{\tilde{u}}_{0}
$$


This asymptotic coordinate transformation is associated to a corresponding null tetrad transformation; which in the leading orders is given by

$$
\begin{gathered}
\tilde{\ell}=d \tilde{u}=\dot{\tilde{u}}_{0} d u+\tilde{u}_{0 \zeta} d \zeta+\tilde{u}_{0 \bar{\zeta}} d \bar{\zeta}+O\left(\frac{1}{r}\right) \\
=\dot{\tilde{u}}_{0} \ell-\frac{\partial_{V} \tilde{u}_{0}}{r} \bar{m}-\frac{\bar{\partial}_{V} \tilde{u}_{0}}{r} m+O\left(\frac{1}{r}\right) . \\
\tilde{n}=\frac{\partial}{\partial \tilde{u}}+O\left(\frac{1}{r}\right)=\frac{1}{\dot{\tilde{u}}_{0}} \frac{\partial}{\partial u}+O\left(\frac{1}{r}\right) \\
=\frac{1}{\dot{\tilde{u}}_{0}} n+O\left(\frac{1}{r}\right), \\
\tilde{m}=\frac{\sqrt{2} \tilde{P}}{\tilde{r}} \frac{\partial}{\partial \tilde{\zeta}}+O\left(\frac{1}{r^{2}}\right)=\frac{\sqrt{2} P_{0} \tilde{V} w}{r}\left(-\frac{\tilde{u}_{0 \zeta}}{\dot{\tilde{u}}_{0}} \frac{\partial}{\partial u}+\frac{\partial}{\partial \zeta}\right)+O\left(\frac{1}{r^{2}}\right) \\
=-\frac{\tilde{V}}{2} P_{0} \tilde{V} w \\
r
\end{gathered}
$$

since the metric expressed in terms of the new null tetrad must coincide with the metric expressed in terms of the original null tetrad, it is deduced that

$$
\tilde{V}=\frac{V}{w}=\frac{V}{\dot{\tilde{u}}_{0}}
$$

therefore

$$
\tilde{m}=m-\frac{\partial_{V} \tilde{u}_{0 \zeta}}{r \dot{\tilde{u}}_{0}} n+O\left(\frac{1}{r^{2}}\right)
$$

The null tetrad transformation equations can be used to write the leading order transformation relations for the spinor dyad associated to the null tetrad[7]; namely

$$
\begin{gathered}
\tilde{o}^{A}=\sqrt{\dot{\tilde{\tilde{u}}}_{0}}\left(o^{A}-\frac{\partial_{V} \tilde{u}_{0}}{r \dot{\tilde{u}}_{0}} \iota^{A}\right), \\
\tilde{\iota}^{A}=\frac{1}{\sqrt{\dot{\tilde{u}}_{0}}} \iota^{A} ;
\end{gathered}
$$

which implies that the regular dyad at future null infinity is given by

$$
\begin{gathered}
\hat{\tilde{o}}^{A}=\tilde{\Omega}^{-1} \tilde{o}^{A}=\frac{r}{w} \sqrt{\dot{\tilde{u}}_{0}}\left(o^{A}-\frac{\partial_{V} \tilde{u}_{0}}{r \dot{\tilde{u}}_{0}} \iota^{A}\right)=\frac{1}{\sqrt{\dot{\tilde{u}}_{0}}}\left(\hat{o}^{A}-\frac{\partial_{V} \tilde{u}_{0}}{\dot{\tilde{u}}_{0}} \hat{\iota}^{A}\right), \\
\hat{\tilde{\iota}}^{A}=\tilde{\iota}^{A}=\frac{1}{\sqrt{\dot{\tilde{u}}_{0}}} \hat{\iota}^{A} .
\end{gathered}
$$


We can now easily calculate the component $\Psi_{2}$ of the Weyl tensor, in leading order, with respect to the new null tetrad, obtaining

$$
\tilde{\Psi}_{2}^{0}=\tilde{\Omega} \Psi_{A B C D} \hat{\tilde{O}}^{A} \hat{\tilde{O}} B \hat{\tilde{\iota}}^{C} \hat{\tilde{\iota}}^{D}=\frac{1}{\dot{\tilde{u}}_{0}^{3}}\left(\Psi_{2}^{0}-\frac{2 \partial_{V} \tilde{u}_{0}}{\dot{\tilde{u}}_{0}} \Psi_{3}^{0}+\frac{\left(\partial_{V} \tilde{u}_{0}\right)^{2}}{\dot{\tilde{u}}_{0}^{2}} \Psi_{4}^{0}\right) ;
$$

where in our case we have

$$
\Psi_{3}^{0}=\widetilde{\partial}_{V} \sigma_{0}^{\prime}-\overline{\mathrm{\partial}}_{V} \rho_{0}^{\prime}=-\frac{1}{2} \overline{\mathrm{\partial}}_{V_{R T}} K_{V_{R T}}+O(\lambda),
$$

and

$$
\Psi_{4}^{0}=\dot{\sigma}_{0}^{\prime}-2 U_{00} \sigma_{0}^{\prime}-\overline{\widehat{\partial}}_{V}^{2} U_{00}=\bar{\varlimsup}_{V_{R T}}^{2} \frac{\dot{V}_{R T}}{V_{R T}}+O(\lambda)
$$

where

$$
\sigma_{0}^{\prime}=\lambda\left(\frac{\dot{V}_{R T}}{V_{R T}} \bar{\sigma}_{0}-\dot{\bar{\sigma}}_{0}\right)
$$

and

$$
\rho_{0}^{\prime}=\frac{1}{2} K_{V}
$$

Let us consider a first order transformation generated by

$$
\tilde{u}_{0}=u-\lambda \gamma(u, \zeta, \bar{\zeta})
$$

then one has

$$
\begin{aligned}
\tilde{\Psi}_{2}^{0} & =-\left[M_{0}+\lambda\left(\tilde{M}_{1}+i \tilde{\mu}\right)\right] \\
& =\frac{1}{(1-\lambda \dot{\gamma})^{3}}\left(-\left[M_{0}+\lambda\left(M_{1}+i \mu\right)\right]-\lambda \partial_{V_{R T}} \gamma \bar{\partial}_{V_{R T}} K_{V_{R T}}\right) ;
\end{aligned}
$$

where it is important to note that the transformation law for $\sigma_{0}$ is

$$
\lambda \tilde{\sigma}_{0}=\lambda \sigma_{0}-\lambda \check{\partial}_{V_{R T}}^{2} \gamma
$$

and therefore, since $\gamma$ is real, it can be seen from equation (118) that

$$
\tilde{\mu}=\mu \text {. }
$$

Then, one concludes that

$$
\tilde{M}_{1}=3 M_{0} \dot{\gamma}+M_{1}+\widetilde{\partial}_{V_{R T}} \gamma \overline{\widetilde{\partial}}_{V_{R T}} K_{V_{R T}}
$$

where it is observed that the left hand side is real, and the first two terms of the right hand side are also real; therefore for this equation to make sense one should have the last 
term also real, which at first sight is not obvious. In order to check this let us note that for any quantity $H$ of spin weight $s$ one has

$$
\varlimsup_{V} \overline{\mathrm{\partial}}_{V} H-\overline{\widehat{\partial}}_{V} \varlimsup_{V} H=-s K_{V} H
$$

then, taking $H=\mathrm{\partial}_{V} \gamma$ one has $s=1$ and

$$
\check{\partial}_{V} \bar{\partial}_{V} \partial_{V} \gamma-\bar{\partial}_{V} \check{\partial}_{V}^{2} \gamma=-K_{V} \check{\partial}_{V} \gamma
$$

so that acting with $\overline{\mathrm{\partial}}_{V}$ on the last expression one obtains

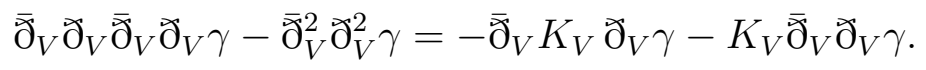

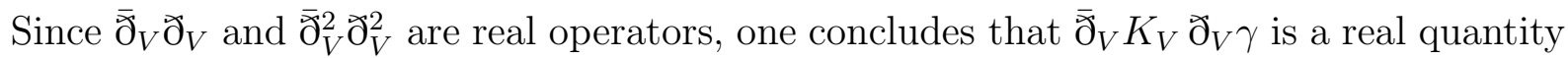
for any $V$.

Coming back to equation (147) we observe that given $M_{1}(u, \zeta \bar{\zeta})$ and $V_{R T}(u, \zeta, \bar{\zeta})$ it is possible to chose $\gamma(u, \zeta, \bar{\zeta})$ such that $\tilde{M}_{1}$ acquires any value that we want.

Alternatively, from equation (132) we have

$$
\tilde{V}=V_{R T}+\lambda\left(V_{\lambda}+\dot{\gamma}\right)
$$

therefore we could also choose $\gamma$ in order to give to $\tilde{V}_{\lambda}$ any desired value.

Summarizing, equations (123), (117) and (119) are the evolution equations for the asymptotic quantities $\Psi_{0}^{0}, \Psi_{1}^{0}$ and $V_{R T}$ respectively. And, equation (120) can be understood as the evolution equation for $V_{\lambda}$ if $M_{1}$ is known; alternatively, it can be understood as the evolution equation of $M_{1}$ if $V_{\lambda}$ is given. As it was mentioned previously, the function $\sigma_{0}$ is free data; which contributes to the description of the structure of the sources and to outgoing radiation.

\section{$5 \quad$ Final comments}

In subsection 3.1 we have presented in full detail the algebraically special vacuum solutions in a new frame. The gauge freedom of these solutions is discussed in subsection 3.2.

The algebraically special perturbations of RT spacetimes, studied in section 3.3, are shown to contain divergent behavior in the asymptotic future, and therefore they do not seem to provide with an appropriate model for the description of a binary collision. This result contrasts with the usual treatments of perturbations of black holes. For example in the study of gravitational perturbations of the Kerr geometry, one normally assumes perturbations which, with respect to the Boyer Lindquist coordinates, have a $t$ and $\phi$ dependence given by $e^{i(\hat{\Sigma} t+m \phi)}[3]$; where $\hat{\Sigma}$ is a constant considered "mostly real and positive" [4]. However it is interesting to note that equation (7.286) of reference [3] reads

$$
\mathcal{C}=D-12 i \hat{\Sigma} M
$$

where $D$ is a positive real constant, $M$ the mass of the spacetime and if particularized to the case of algebraically special perturbation; namely $\Psi_{0}=0$, then the complex constant 
$\mathcal{C}$ must be zero; which indicates that $e^{i(\hat{\Sigma} t+m \phi)}$ has an exponential divergent behavior for late times, as we have found.

One could also ask, how is that this behavior does not appear in the quasi-normal modes study of Schwarzschild black-hole; and the answer is that the very definition of quasi-normal modes excludes the exponentially growing $\Im m \hat{\Sigma}<0$ cases $[1$. It is probably worth while to remark that the quasi-normal modes can not describe the RobinsonTrautman geometry as a perturbation of the Schwarzschild metric.

In relation to the general perturbations of the RT spacetimes, we have shown in section 1 that they provide with a suitable family of spacetimes for the discussion of the estimate of the total energy radiated in the collision of two black holes with orbital angular momentum.

In a future work we will apply this construction to the calculations of the mentioned estimates.

\section{Acknowledgments}

We acknowledge support from Fundación YPF, SeCyT-UNC, CONICET and FONCYT BID 802/OC-AR PICT: 00223.

\section{References}

[1] P. Anninos and S. Brandt. Head-on collision of two unequal mass black holes. Phys.Rev.Lett., 81:508, 1998.

[2] P. Anninos, D. Hobill, E. Seidel, L. Smarr, and W.-M. Suen. Collision of two black holes. Phys.Rev.Lett., 71:2851, 1993.

[3] S. Chandrasekhar. An introduction to the theory of the Kerr metric and its perturbation. In General Relativity, An Einstein Centenary Survey. Cambridge University Press, Cambridge, 1979. Edited by S.W. Hawking and W. Israel.

[4] S. Chandrasekhar. The Mathematica Theory of Black Holes. Oxford University Press, 1992.

[5] S. Dain, O. M. Moreschi, and R. J. Gleiser. Robinson-trautman geometries and the photon rockets. Class. Quantum Grav., 13(5):1155-1160, 1996.

[6] S. Frittelli and O. M. Moreschi. Study of the Robinson-Trautman metrics in the asymptotic future. Gen. Rel. Grav., 24:575, 1992.

[7] R. Geroch, A. Held, and R. Penrose. A space-time calculus based on pairs of null directions. J. Math. Phys., 14:874-881, 1973.

[8] D. Kramer, H. Stephani, MacCallum, and E. Herlt. Exact solutions of Einstein's field equations. Cambridge University Press, Cambridge, 1980. 
[9] O. M. Moreschi. On angular momentum at future null infinity. Class. Quantum. Grav., 3:503-525, 1986.

[10] O. M. Moreschi. General future asymptotically flat spacetimes. Class. Quantum Grav., 4:1063-1084, 1987.

[11] O. M. Moreschi. Supercenter of mass system at future null infinity. Class. Quantum Grav., 5:423-435, 1988.

[12] O. M. Moreschi. Total energy radiated in the head-on black hole collision with arbitrary mass ratio. Phys. Rev. D, 58:084018, 1999.

[13] O. M. Moreschi and S. Dain. Estimates of the total gravitational radiation in the head-on black hole collision. Phys. Rev. D, 53(4):R1745-R1749, 1996. Rapid Communication.

[14] O. M. Moreschi and S. Dain. Rest frame system for asymptotically flat space-times. J. Math. Phys., 39(12):6631-6650, 1998.

[15] O. M. Moreschi, A. Perez, and L. Lehner. Total energy radiated for non head-on binary black hole collisions. in preparation, 2001.

[16] A. Perez and O. M. Moreschi. Characterizing exact solutions from asymptotic physical concepts. submited for publication, http://xxx.lanl.gov/ps/gr-qc/0012100, 2000.

[17] I. Robinson and R. Trautman. Some spherical gravitational waves in general relativity. Proc. R. Soc. A, 265:463-473, 1962. 